\title{
Analysis on Safety Measures of Substation Maintenance
}

\author{
WANG Ji-wen, ZHANG Hai-feng, FANG Yi-guang, WEN Zhi-ke, SHAO Gui-wei, GUO Da-yong
}

China Electric Power Research Institute, Wuhan, Hubei, 430074

Abstract: As an important node in the power grid, substation plays a very important role in the whole power grid. The equipment operation of the substation is carried out in order to timely and effectively detect the operation status of the equipment, find the latent fault of the equipment, and the maintenance of the substation is necessary for safe operation. However, there are some safety problems in the operation of the substation, which need to analyze the status quo of its safe operation and formulate the corresponding improvement measures. Based on this, this article on the substation maintenance work safety measures for a brief analysis, hoping to provide for future reference.

Key words: substation; maintenance; safety measures

\section{The importance of substation maintenance safety research}

In the substation operation process, which will be subject to many factors, including environmental factors and human factors, thus affecting the safety operation of substations, and even a variety of failures. Therefore, we must strengthen the daily maintenance of substation equipment, a variety of equipment for regular maintenance. In the substation equipment maintenance site there will be more influencing factors that we need to analyze, find out the dangerous points and then develop appropriate technical measures to improve. In the process of analysis and research, we should fully consider the various environmental changes and risk factors faced by maintenance personnel, and cannot affect the maintenance work. Therefore, it is very important to do a good job in the safety work of substation maintenance.

\section{Substation equipment maintenance content}

\subsection{Bus repairment}

The $10 \mathrm{kV}$ side switchgear is connected to the low side of the main transformer of the station via a tubular insulated conductor, and the tubular insulated conductor is used as a $10 \mathrm{kV}$ bus. This type of bus for the hollow conductor, insulation for the sealed shield, the main insulation material with excellent performance and stability of PTFE. But when it is used as a long distance contact, the joint installation process is more difficult. In the case of a large stone station, for example, the installer performs heat shrinkage at the pipe joint connection, and the process does not regulate

Copyright (C) 2017 WANG Ji-wen et. al.

doi: http://dx.doi.org/10.18686/esta.v4i1.34

This is an open-access article distributed under the terms of the Creative Commons Attribution Unported License

(http://creativecommons.org/licenses/by-nc/4.0/), which permits unrestricted use, distribution, and reproduction in any medium, provided the original work is properly cited. 
the formation of the electrochemical reaction causing discharge. For the maintenance of this new bus in the form of maintenance, according to the actual experience in recent years, in addition to the construction process in addition to the quality of the process of supervision, combined with daily visits to carry out radio frequency discharge waveform detection can be covered to monitor the cable trench, the operation of the tube in the switchgear, so that early detection of hidden dangers.

Figure 1 new bus

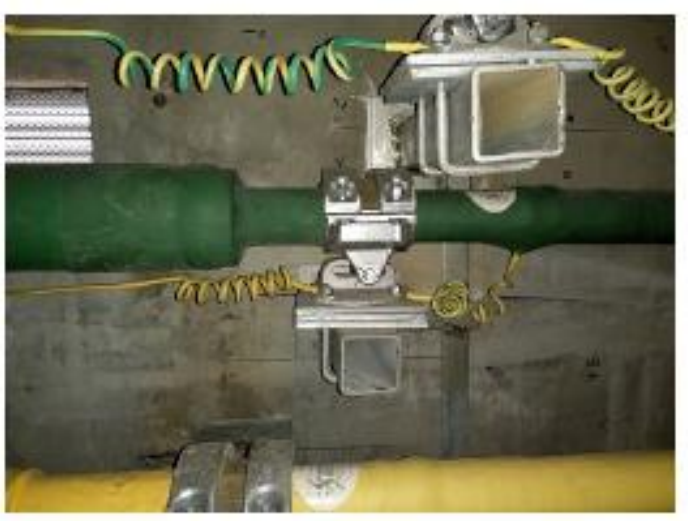

\subsection{Overhaul circuit breakers}

In the substation equipment maintenance, the circuit breaker maintenance is also very critical, more common faults are circuit breaker fire, abnormal sound, internal overheating, malfunction and refusal and so on. Circuit breaker failure occurs, mainly because the battery under voltage, the coil layer between the short circuit, the second wiring is wrong, the coil low voltage failure, the DC voltage is too low or too high and poor control loop, short circuit, as shown in Picture 2. Resulting in more reasons for the malfunction of the circuit breaker, the more common causes of misuse, the power circuit because of two points of insulation or grounding failure lead to failure, the transformer polarity and change ratio in the link process may be wiring errors. In the event of a fault in the circuit breaker, it is necessary to carry out an in-depth analysis according to the actual situation of the fault and the cause of the fault. When the circuit breaker leaps, it is necessary to check the action of the circuit breaker. If the protection action occurs improperly, it is necessary to close the disconnector of the reject circuit breaker, and then ensure that the route circuit is normal. If it is not for the reasons for the closure, you need to cut off all the lines, and then one by one check, troubleshooting reasons.

Figure 2 Circuit breaker structure diagram

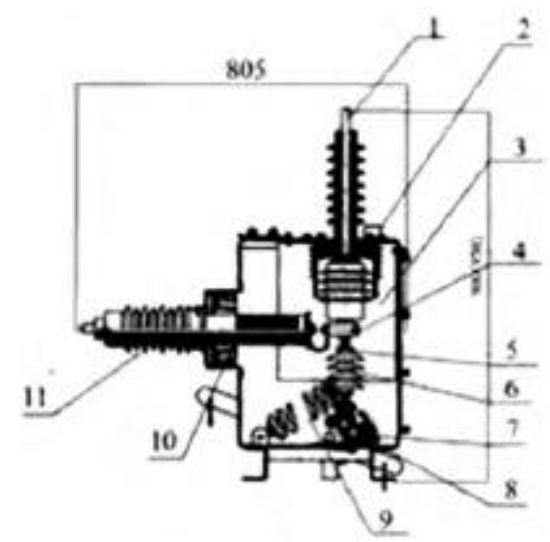

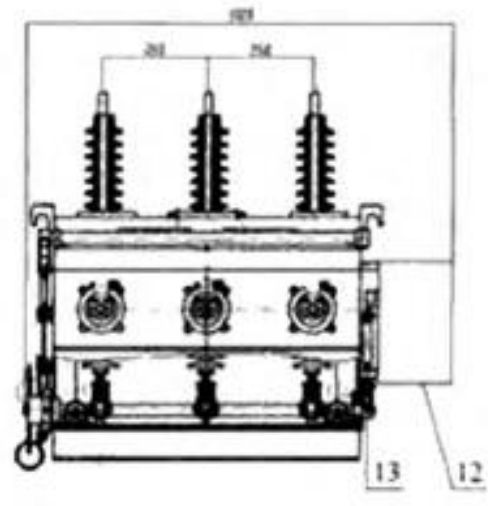


1-Conductor rod insulation sleeve assembly; 2- Vacuum interrupter; 3- Insulated isolation cover; 4-Conductive clip; 5Soft link; 6- Insulated rod; 7- Axis; 8- Shell; 10- Current transformer; 11 - casing; 12 - Operating mechanism; 13 Drive structure; 14 - Voltage transformer.

\subsection{Check the disconnector}

In the substation operation, the isolation switch is the most frequently used equipment, disconnector is also one of the important causes of failure. Usually if the disconnector is improperly installed, or in the installation process without grinding the contact surface, there is no reasonable contact with aluminum and copper contacts, the latter part of the operation may be disconnected switch contact problems, so that the local temperature of the terminal too high. In addition, if the isolation switch design is unreasonable, it will make the contact temperature abnormal. Thus, the common failure of the disconnector is the contact surface temperature is too high, causing overheating. Maintenance personnel need to isolate the common types of faults, fault location, in the manufacturing and installation process to strictly control, using a more mature production process, the structure and connection of the various parts of the scientific design to ensure its performance and running quality.

\subsection{Electronic transformer}

Electronic transformers will voltage and current acquisition of digital, collected data through the fiber optic network for different devices to provide a unified amount of electricity, so as power plant information collection, analysis, sharing lay the foundation. Different from the traditional electromagnetic transformer, electronic transformer without ferromagnetic resonance problem, high precision measurement, high and low voltage side of the electrical isolation, small size, the collected data through the optical fiber transmission, greatly improving the reliability of the automation system, simplifying the wiring. Although there are many differences with the traditional transformer, but in the maintenance, the safety of isolation and traditional substation ideas are more consistent. Maintenance should first disconnect the fiber connection between the modules, and then through a special calibrator for testing, routine testing items generally have electrical unit power check, fiber loop and marking inspection.

\section{Problems in substation maintenance work}

First, some substation repair technology level is too low, the lack of appropriate professional and technical, in the maintenance process using a series of non-standard operation, in the actual maintenance process also ignored the importance of protective measures. As the maintenance work is a long and tedious technical work, maintenance personnel tend to produce boredom, the enthusiasm of the work is not high, there is a certain degree of freedom in the work, and the work is not careful and meticulous, prone to sloppy situation. The requirements are not strict enough, cannot consciously regulate their own behavior, the lack of appropriate safety awareness, did not recognize the importance of maintenance work on the power plant site equipment is not familiar enough, and often habitually use personal work experience in the power station. Check the work, with their own inertia thinking work, resulting in blind operation, in the usual maintenance of the equipment during the work is not in place, to the future operation of the equipment buried the hidden dangers.

Second, the substation management system, there are some loopholes, the lack of scientific, normative substation management, maintenance of the relevant system is not perfect. The management system is not perfect, the lack of safety management concept and the management system cannot be fully implemented, found that the security risks are not timely, the level of power plant managers and lack of capacity, the internal maintenance of the relevant maintenance personnel are not in place. Substation maintenance work of the specific operational procedures, maintenance operators 
have not been subject to institutional constraints, resulting in the relevant personnel in the maintenance work when the randomness and blindness, the equipment maintenance is not thorough, will leave a security risk, overhaul the equipment, which will cause excessive waste of funds. In the substation management cannot strictly implement the responsibility system, for each work project, the job is not really responsible for the implementation of the final substation internal departments of the corresponding job content and accident responsibility, do not have a clear understanding, to a certain extent caused maintenance personnel on the work of the sense of responsibility is not strong. Third, the substation equipment aging and other defects, there is a threat of security abnormalities, the traditional equipment maintenance methods in the equipment maintenance process lack of targeted, cannot be completely flawed in the power equipment to eliminate, and may also increase the equipment of the security risks, exacerbated the instability of power equipment, not only increased the maintenance staff of the workload, to the power companies caused unnecessary waste of funds, increase the burden of power companies, and will lead to frequent power outages situation, affecting the normal life of the masses demand for electricity, maintenance personnel lack of scientific understanding of equipment maintenance, easily lead to the occurrence of electrical accidents.

\section{Improve the substation maintenance work safety measures and methods}

\subsection{Strengthen the electrical equipment maintenance management system}

For the substation in the electrical equipment maintenance management system is not perfect, should strengthen the management system construction, develop strict management system, and increase management efforts to strengthen the safety awareness of maintenance personnel to improve their work enthusiasm and the importance of the work, do good electrical equipment maintenance work. The implementation of job responsibility system, strengthen the sense of responsibility of maintenance personnel, and enhance their sense of responsibility to prevent problems when the transfer of responsibility to each other.

\subsection{Safety measures for maintenance tests}

(1) According to the dispatch instruction will protect the signal, and the corresponding GOOSE export platen removed, so that the misuse of the maintenance device trip phenomenon can be logically prevented.

(2) For a series of important protection equipment such as the bus differential failure in protection and the main transformer three-way jump, it is necessary to remove the transmission plate of the maintenance equipment only when the protection signal is changed, and the control plate remove, so even if the logical exception occurred in the maintenance of equipment, running the device will not happen to false tripping phenomenon.

(3) Disconnect the GOOSE interface on the service unit so that it can perform a good physical isolation. The maintenance personnel can also do this for the relevant operations.

4.3 Safety isolation measures when handling the unit

\subsubsection{Electronic Transformers}

Operation safety policy: Under the premise of scheduling permission, should be associated with the protection device outlet pressure plate, AC circuit pressure plate all out, the merger unit maintenance plate smoothly put away. If the maintenance program needs to be intermittent power outage, after the maintenance interval to exit after the bus differential protection can still work. If the exception occurs in the module, the abnormality occurs in the merging unit, the merging unit should be disconnected from the optical fiber with the protection and control value. Unit outside has a lot of fiber, it is best not to merge the optical fiber in the demolition of the operation, in addition to the location of the choice of fiber, it should preferably be on the front module or at the protective area. 


\subsubsection{Relay protection device}

Operation safety policy: Under the premise of scheduling permission, to remove the GOOSE outlet plate and accept the pressure plate, and then put the device to repair the pressure plate, the device maintenance pressure plate put up for security protection. Maintenance of security policy: Disconnect the protective fiber between the protection and the intelligent terminal to protect the network with the switch network fiber should be disconnected

\subsubsection{Switches}

To run a security policy: disable the affected protection. Overhaul security policy: the switch on the device fiber and cascade fiber are disconnected.

\subsection{To complete the repair after the repair}

After the equipment has been inspected, the corresponding test items should be used to verify that the installation of the equipment is correct. The following tests should be carried out before the three restoration equipment:

\subsubsection{Relay protection device}

Configuration check: Make sure that the protection output GOOSE trip message is configured to be consistent with the original protection. Static test: the protection of the sampling value of the accuracy and protection of the state to check.

\subsubsection{Switches}

Configuration check: According to the switch configuration list to check the configuration of the switch, the specific content to include the management address, static VLAN, time parameters.

\subsubsection{Intelligent operation box}

Configuration Check: The GOOSE message configuration of the smart end should be consistent with the original configuration.

\subsection{Do a good job of substation maintenance personnel training}

Do a good job of electrician maintenance personnel technical training, enrich their expertise, which for improving the professional skills of maintenance personnel, fully aware of and understand the safety hazards at the maintenance site and the risk level has a very important role, through the existence of security risks can be judged may cause the type of accident. So it can improve the efficiency of substation equipment maintenance, while trying to rule out the maintenance process of the existence of risk factors. For the training of maintenance personnel, mainly related to the safety performance of substation equipment and the characteristics of the application of various components of equipment, safety knowledge training, how to deal with the accident, as well as maintenance techniques and methods of application and so on. Relevant leaders and responsible personnel to fully implement and implement the technical training of maintenance personnel, to develop its ability to learn by analogy.

\subsection{Strengthen the safety management of substation facilities}

In order to reduce the security risks caused by equipment reasons, in the early stage of substation infrastructure construction must do all aspects of management, in the choice of electrical equipment to take into account the parameters before and after the match, the performance requirements for the equipment should also meet the actual situation. In the equipment acceptance to follow the appropriate norms to prevent the occurrence of accidents due to equipment reasons. In the daily work of the operation staff should also strengthen the inspection work of the equipment, always pay attention to the work of the equipment to ensure the safe operation of electrical equipment. During the inspection process, the operation of the equipment can be checked by the sound, vibration state, surface temperature and so on. For those who use a longer period of time should strengthen the inspection of the equipment, increase the intensity of inspection to prevent failure. For the inspection of electrical equipment can be divided into regular and occasional inspection, can be combined with substation power equipment, the specific circumstances, a reasonable match to ensure the safe operation of substations. 
4.7 Identify and prevent the risk factors around the maintenance equipment

In the process of substation equipment maintenance, we must first on the power equipment around the existence of the security risks for effective identification, and then develop appropriate preventive measures and methods to prevent the risk factors in the environment into maintenance work safety hazards. In the process of maintenance of power equipment, the existence of the risk factors are uncertain and the characteristics of change, before the maintenance. The

first maintenance personnel to hand over technology, for possible security risks in advance to do a lot of preventive measures to reduce the uncertainty of the risk factors to the construction of the impact.

\section{Conclusion}

For the substation repair site, in order to improve the safety of the maintenance site, we must first analyze the possible security risks, in the usual work to constantly sum up experience, improve their professional skills level, in strict accordance with the operating process for maintenance work. For the safe operation of substations to create favorable conditions.

\section{References}

1. Huang Dong Xing. Substation Operation Maintenance and Maintenance Technology [J]. Intelligent City, 2016,01: 66-67.

2. Zhang Cai Xia, Guo Xiang Shu. Brief Description of Substation Maintenance Status and Development Trend [J]. Shandong Industrial Technology, 2016,03: 153.

3. Li Shuai Yuan, Yang Chuan Long, Miao Yu. Analysis of Substation Secondary Equipment State Maintenance [A].

Beijing Chinese and foreign soft information technology research institute. The third century Star Innovation Education Forum Proceedings [C]. Beijing Chinese and foreign soft information Technology Research Institute :, 2016: 1. 